\title{
Tratamiento rehabilitador en las inestabilidades mediocarpianas palmares
}

\section{Conservative Management in Palmar Midcarpal Instabilities}

\author{
Eva Guisasola Lerma10 Francisco Javier Lucas Garcia ${ }^{2}$
}

1 Médico Rehabilitador Quirón Salud Valencia, Valencia, Spain

2 Traumatología Quirón Salud Valencia, Valencia, Spain

Rev Iberam Cir Mano 2019;47:124-130.
Address for correspondence Eva Guisasola Lerma, Médico Rehabilitador Quirón Salud Valencia, Av. de Blasco Ibáñez, 14, 46010 València, Valencia, Spain (e-mail: evaguisa@yahoo.es).

\section{Resumen \\ Palabras clave \\ - inestabilidad mediocarpiana palmar \\ - muñeca \\ - ortesis \\ - propiocepción \\ - fortalecimiento}

Las inestabilidades mediocarpianas son una patología provocada por la descoordinación en el movimiento entre la hilera proximal y distal del carpo. Las más frecuentes son las inestabilidades palmares $(\mathrm{PMCl})$ y suelen ocasionar un dolor y un clunk muy característico con determinados movimientos de la muñeca. Su diagnóstico en ocasiones no es fácil ya que no existen pruebas de imagen diagnósticas específicas. La primera opción de su tratamiento siempre será conservadora a través de rehabilitación. Actualmente no existen estudios randomizados con series amplias en cuanto a resultados de los tratamientos propuestos dado que no es una patología muy frecuente. En este artículo, se propone un protocolo de trabajo en base a la revisión de los estudios biomecánicos existentes hasta el momento actual.

Midcarpal instabilities are a pathology caused by the uncoordination on the move between proximal and distal carpal rows. The most frequent are palmar instabilities (PMCl) and usually cause pain and clunk very characteristic with certain movements of the wrist. Sometimes their diagnosis aren't easy because there aren't specific diagnosed imaging tests. The first option of their treatment will always be conservative through rehabilitation. Currently there are no randomized studies with large series with results of the proposed treatments because is not a frequent pathology. In this article a work protocol is proposed based on the review of existing biomechanical studies up to the present time.

\section{Introducción}

Las inestabilidades carpianas comprenden una serie de lesiones en los ligamentos del carpo que condicionan esencialmente dolor en la muñeca y pérdida de fuerza de prensión palmar. Una muñeca con disfunción carpiana sintomática no va a ser capaz de soportar cargas fisiológicas y va a presentar una cinemática anormal (descoordinación de movimientos) en su arco de recorrido.

Por ello, es importante que sean diagnosticadas de una manera correcta y lo más precoz posible para evitar que evolucionen a una degeneración articular de los huesos del carpo y como consecuencia de ello, a una artrosis temprana.

Las inestabilidades mediocarpianas forman parte del grupo de las INESTABILIDADES CARPIANAS NO DISOCIATIVAS (CNID), es decir, aquellas en que las que existe disrupción entre huesos de las distintas hileras del carpo, proximal y distal. ${ }^{1}$

El término de "inestabilidad mediocarpiana ulnar fue descrito por primera vez por Lichtman en 1981, citando 10 pacientes que presentaban dolor y chasquido durante la desviación ulnar con la muñeca en posición pronada. ${ }^{2}$ received

September 26, 2019

accepted

October 7, 2019
DOI https://doi.org/

10.1055/s-0039-3399517. ISSN 1698-8396.
Copyright ๑ 2019 Thieme Revinter

Publicações Ltda, Rio de Janeiro, Brazil
License terms

c) $(1) \$$ 
Posteriormente se sustituyó el nombre por el de Inestabilidades Mediocarpianas Palmares (PMCI) con el propósito de distinguirlas de las inestabilidades dorsales. ${ }^{3}$ Se describieron los distintos tipos de inestabilidades mediocarpianas dinámicas: las palmares, dorsales, combinadas (dorsales y palmares) y extrínsecas (secundarias a un traumatismo o a una mala alineación carpiana tras una fractura de radio).

De todas las causas que producen PMCI, la más frecuente es por insuficiencia de los complejos ligamentosos palmares piramidal-ganchoso-hueso grande $\mathrm{y}$ del complejo escafoides-trapecio-trapezoide. ${ }^{1}$ (-Fig. 1)

La primera línea de tratamiento en esas inestabilidades va a ser conservador. En el siguiente artículo, se hace una revisión de los distintos tratamientos propuestos y se describe cuál es la tendencia actual en el manejo conservador de ese tipo de lesiones.

\section{Aspectos Biomecánicos}

La articulación mediocarpiana cumple un papel muy importante en los movimientos de la muñeca, por tanto, un mal funcionamiento de esa articulación, podrá influir en la cinemática global del carpo:

Los movimientos de la muñeca se realizan a través de tres componentes rotacionales en tres ejes diferentes: (-Fig. 2)

- El eje transversal nos permite el movimiento de flexoextensión: la articulación mediocarpiana es la responsable del 33\% de extensión de la muñeca y del $60 \%$ de la flexión.

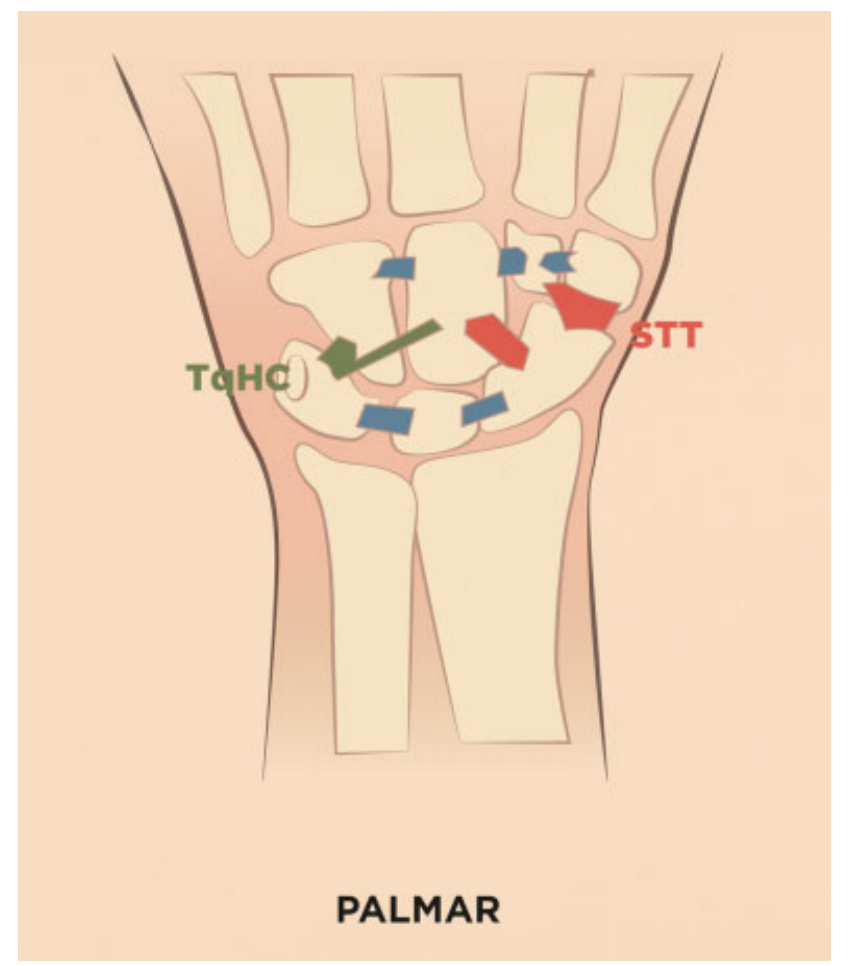

Fig. 1 Las inestabilidades mediocarpianas más frecuentes son por afectación de los ligamentos Piramidal-ganchoso-grande y escafoides-trapecio-trapezoide.

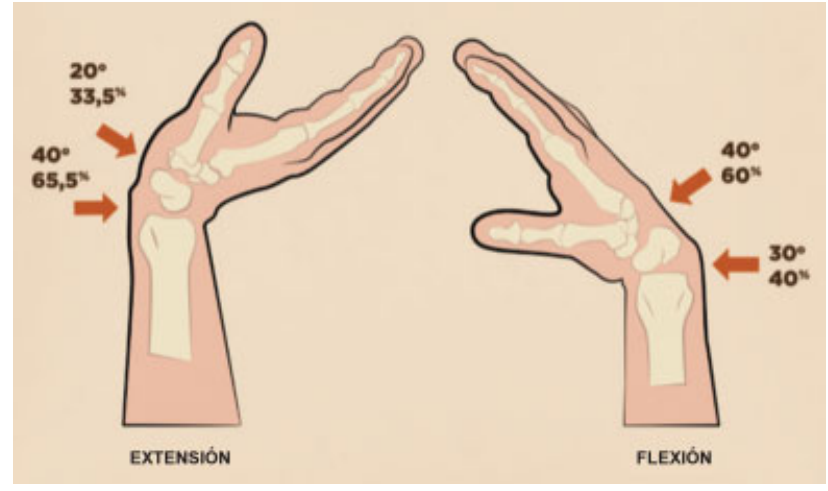

Fig. 2 La articulación mediocarpiana es la responsable del 33\% de la extensión de la muñeca y del $60 \%$ de la flexión.

- El eje sagital nos permite el movimiento de desviación radial y desviación ulnar. $10^{\circ}$ de la desviación radial y $25^{\circ}$ de la desviación ulnar son responsabilidad de la mediocarpiana.

- El eje longitudinal permite el movimiento de pronosupinación y éste se produce mayoritariamente en antebrazo.

También es la responsable de una combinación de movimientos en el plano oblicuo de la muñeca definida como "Dart Throwing Motion" (DTM) que implica el gesto de extensión de muñeca con desviación radial y flexión de la misma con desviación cubital. Movimiento del que forman parte el $90 \%$ de las actividades de la vida diaria en lo que a la articulación de la muñeca se refiere. ${ }^{1}$ Ese concepto de movimiento en el plano oblicuo ha condicionado un cambio en cuanto a la cinemática carpiana se refiere, que inicialmente se consideraba sólo en el plano sagital y transversal. ${ }^{4}$

Bajo una carga axial, con gestos tan cotidianos como realizar la prensa, ejercer un apoyo palmar o coger un peso, los distintos huesos del carpo tienen un comportamiento diferente:

El hueso escafoides, dada su oblicuidad con respecto al eje longitudinal de la muñeca, tiene tendencia a la flexión y pronación apoyado sobre el semilunar. Ello va a condicionar que el hueso trapecio tienda a desplazarse en sentido palmar también.

Sin embargo, el hueso piramidal, por la tracción que ejercen sobre él potentes ligamentos como piramidal-ganchosogrande, tenderá a la extensión y supinación; por tanto, el hueso ganchoso también tiende a desplazarse en sentido dorsal.

Esos movimientos contrapuestos del trapecioy del ganchoso generan un movimiento rotacional o pronador alrededor de la cabeza del hueso grande. Sin embargo, en condiciones normales, ese desplazamiento se va a contrarrestar por:

1. La acción estabilizadora de los estabilizadores primarios, que son los ligamentos piramidal-ganchoso-hueso grande y del ligamento escafoides-trapecio-trapezoide.

2. La acción coordinada de los estabilizadores secundarios. En ese caso, se ha comprobado que una contracción coordinada del extensor carpi ulnaris (ECU) y del flexor carpi ulnaris (FCU) contribuyen también a evitar ese desplazamiento. $^{5}$ 
Cuando pasamos de la posición de desviación radial a desviación ulnar en una muñeca en pronación y no patológica, toda la hilera proximal del carpo rotará de flexión a extensión. Este movimiento será progresivo, suave y sin cambios bruscos y no apreciaremos nada gracias a la acción contrarrestada de los estabilizadores primarios (ligamentos intactos) y secundarios (contracción coordinada del ECU y del FCU). ${ }^{6}$ La contracción aislada del ECU provoca una extensión y pronación de la hilera distal del carpo. Se ha comprobado experimentalmente que seccionando los ligamentos piramido-grande-ganchoso y escafoides-trapecio-trapezoide, solo el efecto del músculo ECU es capaz de contrarrestar la excesiva flexión de la hilera proximal. La contracción aislada del FCU también va a inducir una fuerza sobre el piramidal a través del pisiforme que inducirá la extensión también de la primera hilera. La acción combinada de ambos va a contribuir a una mayor estabilización de la muñeca. ${ }^{7}$

Sin embargo, si una muñeca presenta una inestabilidad mediocarpiana, cuando pasamos de desviación radial a cubital estando ésta en pronación, la hilera proximal permanecerá anormalmente flexionada hasta que se produce la extensión súbita al final del movimiento (resalte visible o audible o "clunk").

Todas esas consideraciones biomecánicas van a ser la base del tratamiento conservador en ese tipo de lesiones.

\section{Mecanorreceptores a Nivel de Los Ligamentos Implicados}

La existencia de mecanorreceptores en los ligamentos palmares de la muñeca fue inicialmente documentada por Petrie y col., en $1997 .^{8}$

En el 2007, Hagert y col. demostraron mediante inmunohistología que los ligamentos dorsales del carpo presentan una gran riqueza de mecanorreceptores, así como el complejo ligamentoso palmar piramido-grande-ganchoso. ${ }^{9}$ Posteriormente demostraron la presencia de reflejos propioceptivos cuya función es la estabilización de la muñeca. ${ }^{10}$

Es decir, la incompetencia de los ligamentos y el fallo en el control propioceptivo son los que van a condicionar la aparición de una inestabilidad mediocarpiana palmar. Por ello será imprescindible que el trabajo propioceptivo esté presente en el tratamiento conservador de ese tipo de lesiones.

\section{Tratamiento Rehabilitador}

Como en todas las inestabilidades carpianas, el tratamiento debe ir encaminado a restablecer el proceso que provoca la pérdida de la estabilidad.

En el caso de las inestabilidades mediocarpianas, la terapia inicial e imprescindible consistirá en restablecer el adecuado feedback propioceptivo entre los mecanorreceptores y los músculos que van a controlar dicha articulación.

Los pilares básicos en los que se va a sustentar el tratamiento son: El uso de una ortesis adecuada, modificación de determinadas actividades y gestos cotidianos que la provocan y ejercicios de fortalecimiento de la articulación mediocarpiana y propioceptivos. ${ }^{11}$ En ocasiones, la infiltración de corticoides a nivel intraarticular puede aliviar el dolor provocado por la inflamación a ese nivel.

El tiempo mínimo de tratamiento considerado son 6-8 semanas y puede llegar a prolongarse hasta 6 meses. Tras las primeras 6-8 semanas deberá reevaluarse al paciente y plantearse tras una detallada exploración clínica la conveniencia de proseguir con el tratamiento rehabilitador si hay una clara mejoría clínica.

Existen casos en que la falta de respuesta a ese tratamiento obliga a plantear un tratamiento quirúrgico. De forma clásica, se han realizado técnicas de reconstrucción del ligamento arcuato volar o los ligamentos radiocarpianos dorsales ${ }^{12} \mathrm{o}$ transferencias tendinosas para estabilizar la fila proximal en los casos más leves y las artrodesis parciales, con la consiguiente pérdida de movilidad, para los casos más graves. En la actualidad, se han desarrollado técnicas artroscópicas como el retensado térmico capsular dorsal ${ }^{13} \mathrm{o}$ la más reciente plicatura dorsal artroscópica ${ }^{14}$.

\section{Uso de Ortesis}

Se confeccionará una ortesis a medida cuya característica esencial será que ejerza una presión volar sobre el pisiforme hacia dorsal y una contrapresión sobre estiloides ulnar. La finalidad de dicha ortesis es contrarrestar la tendencia a la flexión de la hilera proximal.

Es fundamental que el paciente lleve la ortesis durante todo el día al menos 2 semanas, incluso durante el descanso nocturno, permitiéndole retirársela para la higiene diaria y para la realización de los ejercicios varias veces al día.

Las ortesis más comúnmente utilizadas provocan una inmovilización del borde ulnar de muñeca con presión sobre pisiforme y una contrapresión sobre la estiloides cubital, añadiendo una cincha de velcro en la muñeca y otra rodeando el pulgar. ${ }^{15}$ ( - Fig. 3 )

Posteriormente se han ido diseñando nuevas ortesis que intentan evitar la rigidez y la atrofia provocada por la inmovilización permitiendo un arco de movilidad controlado. Entre ellas se encuentran la propuesta por $0^{\prime}$ Brian $^{16}$ (2013) que va a permitir mantener ciertos recorridos articulares en la muñeca $\left(30^{\circ}\right.$ de extensión, $20^{\circ}$ de flexión, $10^{\circ}$ de desviación radial y $20^{\circ}$ de desviación ulnar) (-Fig. 4)

Más recientemente, en 2015, Braidotti y col., proponen la Dart Splint. ${ }^{17}$ Ese dispositivo nos va a permitir la movilidad de la muñeca en el eje oblicuo permitiendo el DTM (Dart Throwing Motion) gracias a dos bisagras, al tiempo que nos va a ejercer las presiones necesarias para la estabilización de la muñeca (-Fig. 5).

En algún caso menos severo o mientras esperamos a la confección de la ortesis, el vendaje mediante Kinesiotaping puede ayudarnos a controlar el dolor inmovilizando el borde ulnar con la venda volarmente desde el pisiforme hacia la zona dorsal presionando sobre estiloides cubital y acabando en el aspecto radial dorsal distal del radio proporcionando soporte en la columna ulnar de la muñeca. 


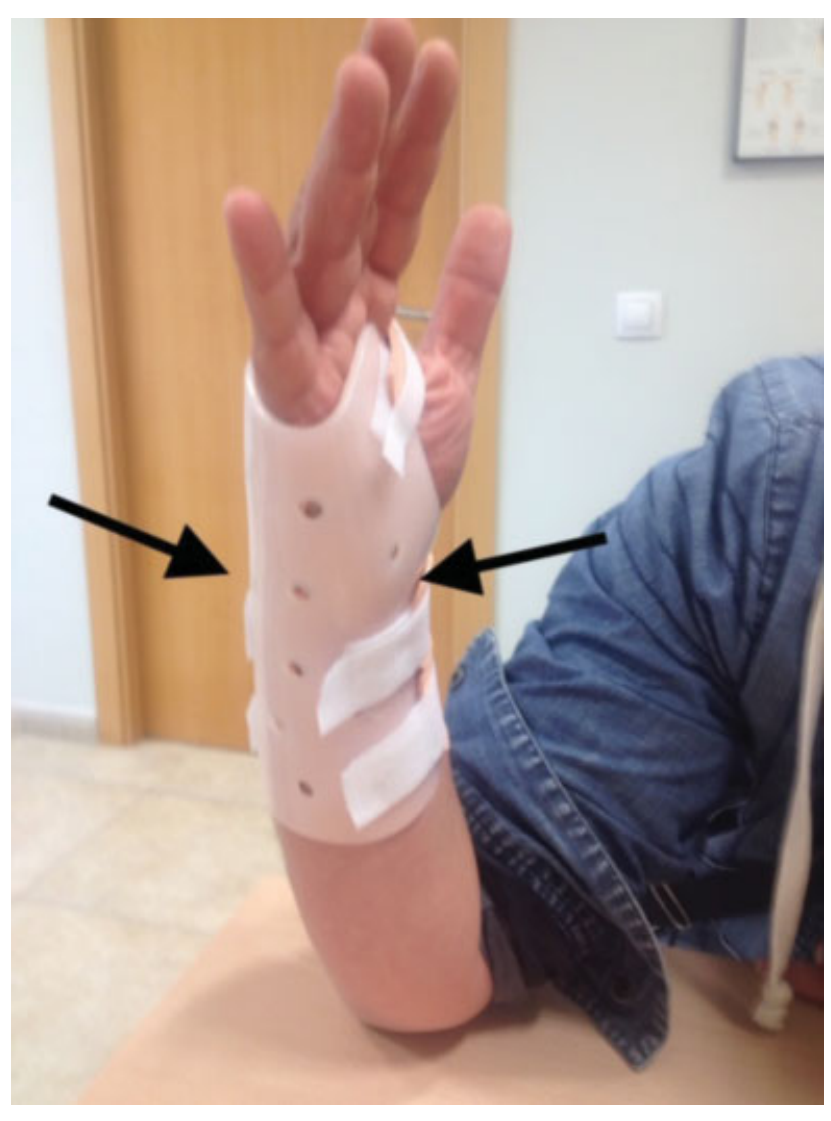

Fig. 3 ortesis clásica de termoplástico. Ejerce una presión volar sobre pisiforme y contrapresión sobre estiloides cubital.

\section{Trabajo Propioceptivo}

La propiocepción es el sentido somático descrito por Charles Scout Sherrington en 1906, para definir las sensaciones provenientes de las áreas profundas del cuerpo, que contribuyen al equilibrio postural y a la estabilidad articular.

En el año 2010, Elisabet Hagert ${ }^{10}$ propone un protocolo de rehabilitación propioceptiva para la muñeca que se ha ido adaptando en base al tipo de patología que se vaya a tratar.

Inicialmente se deberá trabajar el control propioceptivo de las posiciones de la muñeca que consiste en el entrenamiento del sentido de la posición de la misma:

Primero anulando el sentido de la vista intentaremos que reproduzca con la muñeca afecta, exactamente la posición en la que coloquemos pasivamente la no afecta. Eso provocará una estimulación máxima de los receptores articulares.

Posteriormente se entrenará con la terapia en espejo (Mirror Therapy): ese concepto fue presentado por primera vez en el 2005 por Rosen y Lundborg ${ }^{18}$ con la idea de dar ilusión de función a una mano no funcionante. Esa terapia va a permitir la reorganización somatosensorial del córtex cerebral mediante el estímulo propioceptivo de la vista con la que se intentará reproducir una serie de movimientos determinados realizados con la muñeca sana ( - Fig. 6) lo cual va a ayudar a mejorar la coordinación en la muñeca inestable.

\section{Fortalecimiento Muscular - Estabilización Dinámica}

Inicialmente será muy importante instruir al paciente en la corrección postural de su muñeca durante determinadas

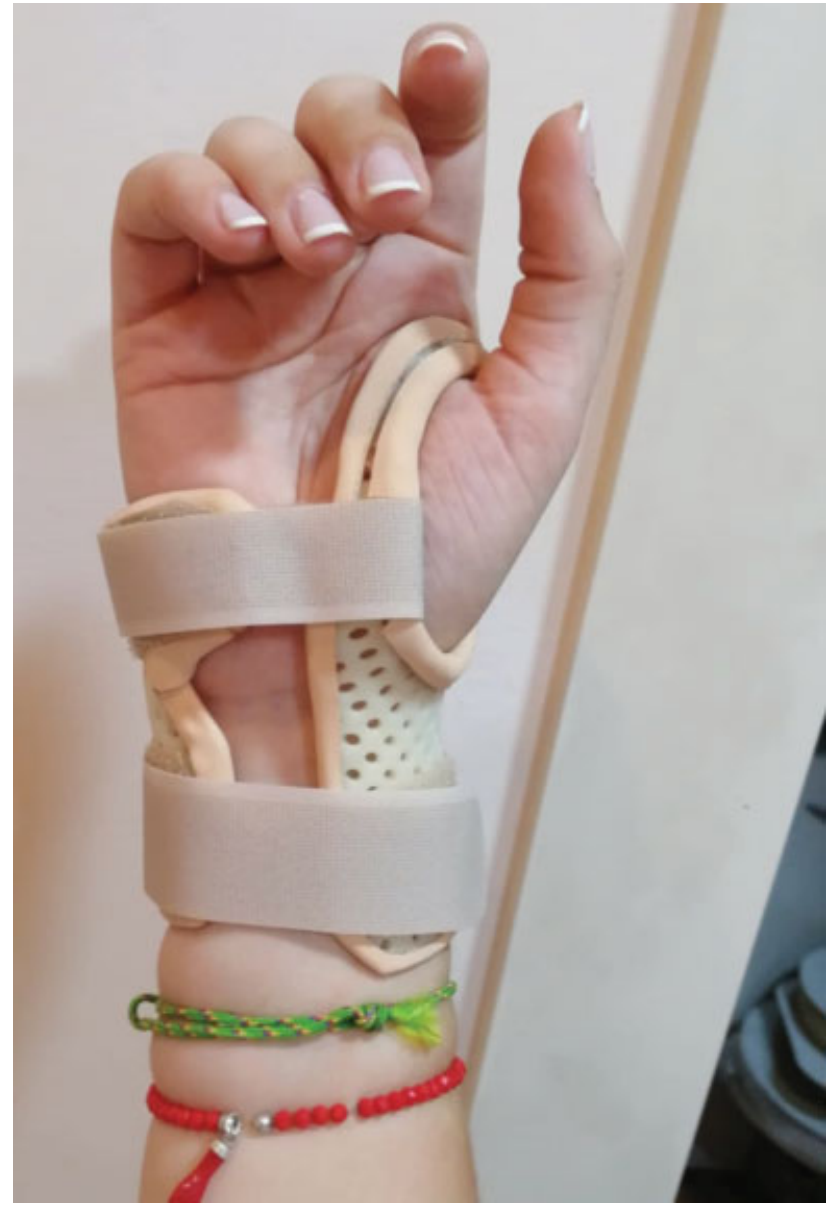

Fig. 4 Ortesis propuesta por O’ Brian. Permite ligera flexo-extensión y desviaciones de muñeca.

actividades con la finalidad de amortiguar o evitar el clunk doloroso (Ej.: posicionamiento en neutro de la muñeca a la hora de realizar algún tipo de levantamiento, vendajes funcionales, etc.). ${ }^{19}$

Se trabajará la activación muscular para compensar la laxitud ligamentosa mediante un programa de ejercicios de fortalecimiento con la finalidad de estabilizar la muñeca a nivel ulnar.

En esa patología, en la que se produce una inestabilidad entre las dos hileras del carpo, deberemos evitar inicialmente:

- Ejercicios que impliquen recorridos articulares repetitivos de muñeca.

- Ejercicios isotónicos tanto concéntricos como excéntricos (que implican recorridos articulares con acortamiento y con alargamiento muscular respectivamente).

- Curls de muñeca con y sin peso (ejercicios que implican poco control).

- Ejercicios de prensa.

- Los distintos grupos musculares que debemos trabajar son dos:

- FCU (Flexor Carpi Ulnaris): Cuya contracción aislada fuerza la extensión del piramidal.

- ECU (Extensor Carpi Ulnaris): Se ha comprobado que es capaz de contrarrestar la excesiva flexión de la hilera 


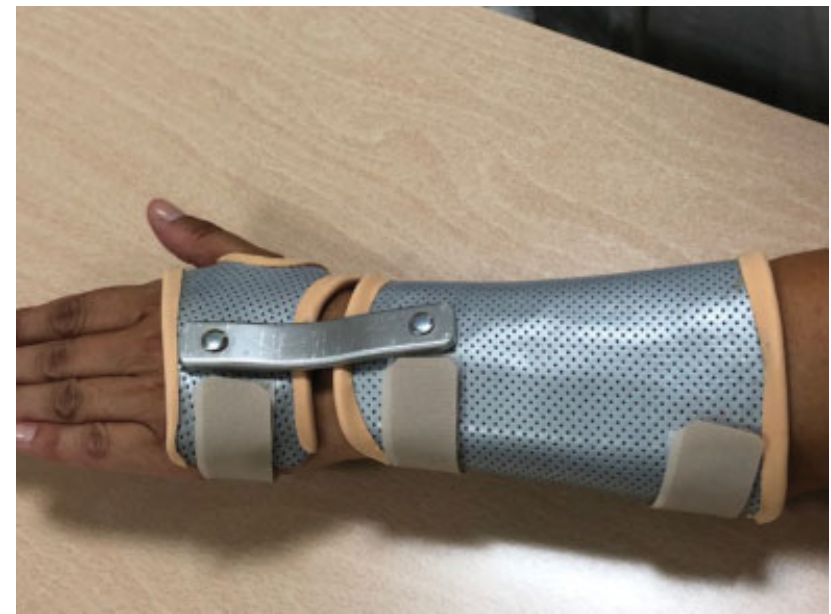

Fig. 5 Dart Splint. Permite el movimiento combinado de extensión y desviación radial y de flexión más desviación cubital.

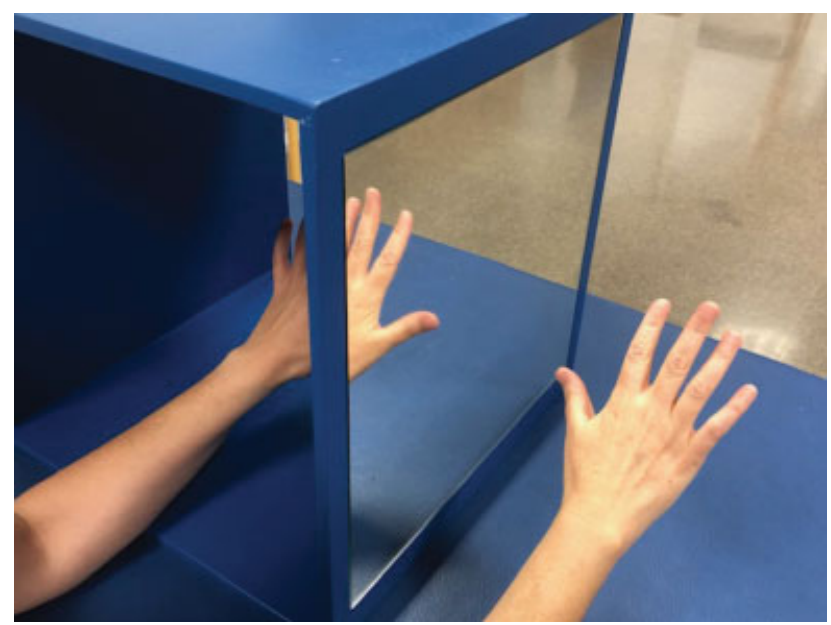

Fig. 6 Terapia en espejo. Control propioceptivo de la posición de la muñeca.

proximal. Es un pronador de la hilera distal y además produce extensión del hueso piramidal.

Se trabajará siempre en isométrico, es decir, la fuerza muscular ejercida no va a superar la resistencia externa ofrecida, no cambia así la longitud del músculo y no habrá por tanto desplazamiento del segmento articular. ${ }^{20}$

Mejor trabajar en co-contracción del FCU y ECU, que contracciones isométricas aisladas: la contracción simultánea de los dos grupos musculares constituye un ejercicio más eficiente y a la vez genera mayor estabilidad de la articulación, dado que la coactivación de ambos músculos nos va a englobar trabajo excéntrico, concéntrico e isométrico. ${ }^{7}$

Se iniciará pues el programa de recuperación funcional instruyendo primero al paciente para tomar control activo de su muñeca. Aprenderán a localizar mediante palpación los músculos que protegen su articulación inestable, cómo aislarlos y tras ello, iniciar un programa de ejercicios isométricos graduales que vayan fortaleciéndolos de una manera suave y controlada.

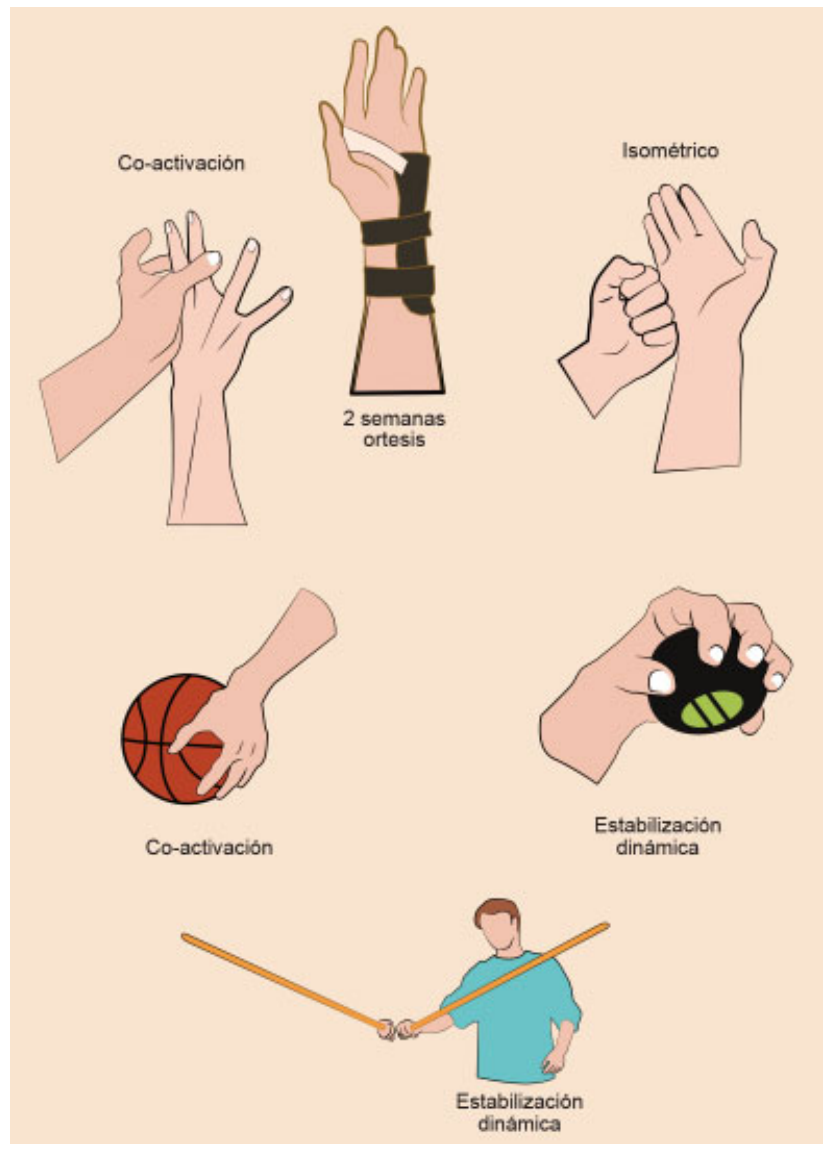

Fig. 7 Ejercicios propuestos. Ortesis, fortalecimiento y ejercicios de estabilización dinámica.

Con las premisas expuestas, los ejercicios con los que se trabaja en clínica y que se proponen son:

Ejercicio isométrico de desviación ulnar. Con la muñeca en posición de supinación (de esa forma disminuye la varianza cubital y ello produce un efecto tensor sobre los ligamentos ulnares), se realiza un movimiento de desviación ulnar contra la resistencia ejercida sobre cara lateral de la mano.

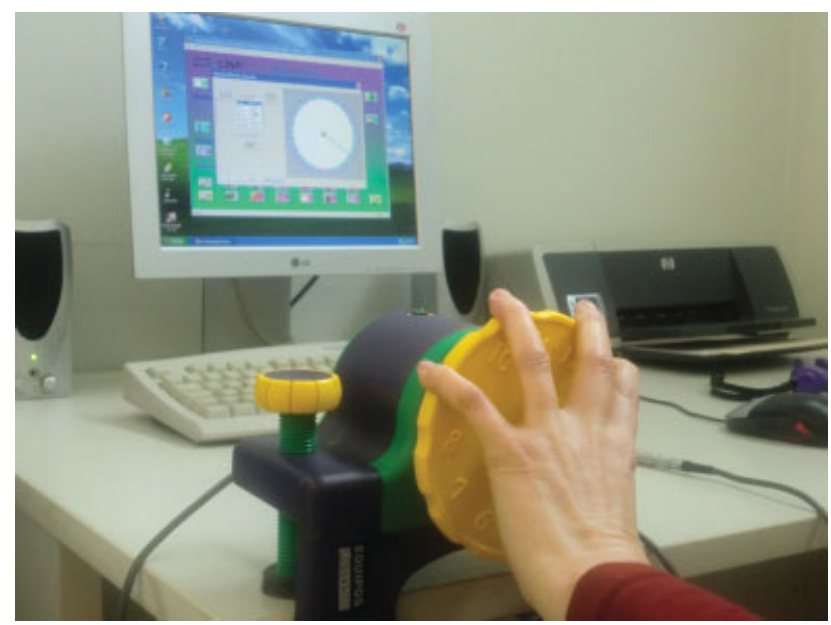

Fig. 8 Biometrics E-Link. Estabilización dinámica mediante activación muscular refleja. 
Tabla 1 Programa rehabilitador propuesto en los casos de inestabilidad mediocarpiana palmar

\begin{tabular}{|c|c|c|}
\hline \multicolumn{3}{|c|}{ TRATAMIENTO CONSERVADOR PMCI } \\
\hline USO DE ORTESIS & $\begin{array}{l}\text { - Ortesis de termoplástico con } \\
\text { presión sobre pisiforme y } \\
\text { contrapresión en estiloides cubital. } \\
\text { - Ortesis que permita mayor } \\
\text { movilidad: O Brian, Dart Splint } \\
\text { - Kinesiotapping }\end{array}$ & $\begin{array}{l}2 \text { semanas ininterrumpidas. Se puede } \\
\text { retirar para el aseo y realización de los } \\
\text { ejercicios. }\end{array}$ \\
\hline REEDUCACION PROPIOCEPTIVA & $\begin{array}{l}\text { Control propioceptivo posiciones de la } \\
\text { muñeca: } \\
\text { - Anulando sentido de la vista } \\
\text { - Terapia con espejo }\end{array}$ & Desde el primer día \\
\hline $\begin{array}{l}\text { FORTALECIMIENTO } \\
\text { ESTABILIZACION DINAMICA }\end{array}$ & $\begin{array}{l}\text { - Isométricos: FCU y ECU } \\
\text { - Co. activación isométrica: } \\
\text { - Estabilización dinámica refleja: } \\
\text { - Estabilización dinámica } \\
\text { consciente: }\end{array}$ & $\begin{array}{l}\text { De } 6 \text { semanas a } 6 \text { meses } \\
\text { Synergi Test } \\
\text { Rodamientos con pelota } \\
\text { Ejercicios con plato y canica } \\
\text { Powerball } \\
\text { Biometrics E-Link } \\
\text { Pliométricos con pelota } \\
\text { Rodamientos con pelota en pared o } \\
\text { mesa. } \\
\text { Reeducar el control de la muñeca de } \\
\text { posición supinada a pronada }\end{array}$ \\
\hline
\end{tabular}

Synergi Test. (test que sirve para provocar la contracción del ECU y FCU y diagnosticar si hay dolor a ese nivel). Apoyando el codo con el antebrazo en supinación, el paciente realiza una abducción de dedos y ejerce una contrarresistencia con los dedos de la otra mano sobre el primero y tercer dedo. Coactivamos simultáneamente el FCU y ECU. (-Fig.7)

Ambos ejercicios habrá que trabajarlos en posición supinada y progresivamente se irán introduciendo en posición neutra y posteriormente en posición pronada conforme se vaya ganando estabilidad.

Ejercicios de estabilización dinámica de la muñeca mediante activación muscular refleja: 7,20 entre ellos existen el balanceo de una canica en un plato, o una pelota de tenis sobre la raqueta (ambos en posición supinada) o el manejo de la "Powerball" o el Biometrics e-Link ${ }^{\mathrm{R}}$. (-Fig. 8) (un paso más avanzado dado que la muñeca ya se encuentra en posición pronada).

La realización de ejercicios pliométricos con pelota blanda (movimientos rápidos de extensión-retracción) del tipo lanzamientos a pared contribuyen también a mejorar la capacidad anticipatoria de los distintos grupos musculares de la muñeca.

Ejercicios de estabilización dinámica consciente: Fortalecimiento de determinados grupos musculares para mejorar la estabilidad de la muñeca:

Rodamientos de pelota contra la mesa o la pared. (otro ejemplo de coactivación muscular).

Reeducación del control de la muñeca de la posición supinada a pronada con pica.

Resumiendo, el programa completo rehabilitador ( - Tabla 1) incluirá unas dos sesiones semanales que deberán ser complementadas con la realización diaria de ejercicios en domicilio. El paciente será debidamente instruido por el terapeuta y no los deberá realizar sólo hasta comprobar su correcta realización.

\section{Conclusiones}

Existen pocos estudios que muestren la evidencia en la efectividad de los distintos programas utilizados en la inestabilidad mediocarpianas. Lichman y col., ${ }^{21}$ reflejaron en su estudio una mejoría de 6 pacientes en una serie de 10 . Wright ${ }^{22}$ habla de una mejoría de un $57 \%$ en su estudio y Pack-Cheong $\mathrm{Ho}^{11}$ de un 55,3\% (9 pacientes) en 2017.

El uso de ortesis no se discute; sería bueno analizar mediante un estudio comparativo qué tipo de ortesis es el más conveniente, si la estática de termoplástico con la presión y contrapresión a nivel ulnar o las últimas propuestas que inmovilizan menos, pero van a permitir iniciar precozmente el trabajo propioceptivo.

En lo que todos los trabajos coinciden es en la conveniencia de iniciarse un tratamiento conservador cuando la inestabilidad mediocarpiana es sintomática. Será imprescindible la reeducación de sus actividades diarias y gestos a nivel laboral y/o deportivo, así como la reeducación propioceptiva y el fortalecimiento de la musculatura estabilizadora. El tratamiento quirúrgico sólo debe ser ofrecido en aquellos casos en los que falle por completo el tratamiento conservador durante un periodo razonable de tiempo.

Conflicto de Intereses

Los autores del trabajo declaran no tener ningún conflicto de intereses. 


\section{Bibliografía}

1 Lichtman DM, Wroten ES. Understanding midcarpal instability. J Hand Surg Am 2006;31(03):491-498

2 Lichtman DM, Schneider JR, Swafford AR, Mack GR. Ulnar midcarpal instability-clinical and laboratory analysis. JHand Surg Am 1981;6(05):515-523

3 Lichtman DM, Bruckner JD, Culp RW, Alexander CE. Palmar midcarpal instability: results of surgical reconstruction. JHand Surg Am 1993;18(02):307-315

4 Moritomo H, Murase T, Goto A, Oka K, Sugamoto K, Yoshikawa H. In vivo three-dimensional kinematics of the midcarpal joint of the wrist. JBone Joint Surg Am 2006;88(03):611-621

5 Esplugas M, Garcia-Elias M, Lluch A, Llusá Pérez M. Role of muscles in the stabilization of ligament-deficient wrists. JHand Ther 2016;29(02):166-174

6 A. Lluch et al. El papel de la propiocepción y el control neuromuscular en las inestabilidades del carpo. Rev Iberoam Cir mano 2015;43(01):70-78

7 Garcia-Elías M. The non-dissociative clunking wrist: a personal view. JHand Surg Eur Vol 2008;33(06):698-711

8 Petrie S, Collins J, Solomonow M, Wink C, Chuinard R. Mechanoreceptors in the palmar wrist ligaments. JBone Joint Surg Br 1997;79(03):494-496

9 Hagert E, García-Elías M, Forsgren S, Ljung BO. Immunohistochemical analysis of wrist ligament innervation in relation to their structural composition. JHand Surg Am 2007;32(01):30-36

10 Hagert E. Proprioception of the wrist joint: a review of current concepts and possible implications on the rehabilitation of the wrist. J Hand Ther 2010;23(01):2-17
11 Ho PC, Tse WL, Wong CW. Palmer Midcarpal Instability: An algorithm of diagnosis and surgical management. JWrist Surg 2017;6(04):262-275

12 von Schroeder HP. Dorsal wrist plication for midcarpal instability. JHand Surg Am 2018;43(04):354-359

13 Higgin RPC, Hargreaves DG. Midcarpal instability: the role of wrist arthroscopy. Hand Clin 2017;33(04):717-726

14 Atzei A, Braidotti F, Hagert E, Luchetti R. Arthroscopic Ligament Plication for Palmar Midcarpal Instability. JWrist Surg 2015;04 (10): 1055

15 Chinchalkar S, Yong SA. An ulnar boost splint for midcarpal instability. J Hand Ther 2004;17(03):377-379

16 O'Brien MT. An innovative orthotic design for midcarpal instability, non-dissociative: mobility with stability. JHand Ther 2013;26(04):363-364

17 Braidotti F, et al. J Hand Ther 2015;xxx:1-6

18 Rosén B, Lundborg G. Training with a mirror in rehabilitation of the hand. Scand J Plast Reconstr Surg Hand Surg 2005;39(02): 104-108

19 Mulders MA, et al. Long-Term Functional Results of a wrist exercise programme. JWrist Surg 2017

20 Harwood C, Turner L. Conservative management of midcarpal instability. J Hand Surg Am 2015; $\cdots: 1$ :8

21 Lichtman DM, Wroten ES. Understanding midcarpal instability. J Hand Surg Am 2006;31(03):491-498

22 Wright TW, Dobyns JH, Linscheid RL, Macksoud W, Siegert J. Carpal instability non-dissociative. J Hand Surg [Br] 1994;19(06): 763-773 